\title{
TWO-DIMENSIONAL LASER DLAGNOSTICS AND MODELING OF COUNTERFLOW DIFFUSION FLAMES
}

\author{
V. SICK, A. ARNOLD, E. DIEßEL, T. DREIER, \\ W. KETTERLE, B. LANGE, J. WOLFRUM, \\ Physikalisch-Chemisches Institut der Universität Heidelberg. \\ Im Neuenheimer Feld 253, 6900 Heidelberg, Federal Republic of Germany
}

\author{
K. U. THIELF
}

Institut für Verfahrenstechnik und Dampfkesselwesen der Universität Stuttgart, Pfaffenwaldring 23, 7000 Stuttgart 80, Federal Republic of Germany

AND

\section{F. BEHRENDT, I. WARNATZ}

Institut für Technische Verbrennung der Universität Stuttgart, Pfaffenwaldring 12, 7000 Stuttgart 80, Federal Republic of Germany and Interdisziplinäres Zentrum für Wissenschaftliches Rechnen der Universität Heidelberg Im Neuenheimer Feld 368, 6900 Heidelberg, Federal Republic of Germany

\section{Introduction}

Laminar counterflow diffusion flames constitute an important basis set for the simulation of turbulent combustion processes. Use of a library of precalculated "flamelets" at different strain rates and fuel/oxidizer mixture compostions, allows the numerical treatment of turbulent flame structures to be reduced-e.g. with a "flamelet model"1-3-to a problem that can be solved within a reasonable amount of time on existing computers. Therefore, there is renewed interest in the study of diffusion flames under a variety of different burning conditions to test and refine the validity of chemistry and transport properties used in modelling these flame structures. Among different types of counterflow diffusion flames the configuration where the flame is established in the forward stagnation region of a cylindrical porous burner offers some inherent advantages. ${ }^{4}$ This type of diffusion flame was studied extensively by Tsuji and coworkers, ${ }^{5-8}$ who also presented an excellent review article covering this topic. ${ }^{4}$ The Tsuji-burner is ideally suited for experimental work for several reasons: strain rate and mixture composition can be adjusted easily, heat conduction problems are minimized because no rigid structures are in contact with the flame, and the flame front can be easily accessed by intrusive and non-intrusive probing techniques. A comparison of data collected by intrusive methods ${ }^{7}$ with numeri- cal modelling results of several groups was presented at the 20th Symposium. ${ }^{9}$

In previous papers, ${ }^{10,11}$ we presented some comparisons between CARS temperature and concentration measurements and numerical modelling results. The disagreement between these results in the low strain rate regime stimulated us to improve and more closely match both the experimental and modelling approaches to this special flame structure.

For a realistic numerical treatment of the flame structure of counterflow diffusion flames, a correct description of the flow field is essential. Earlier models assumed an outer potential flow surrounding the boundary layer. ${ }^{9}$ The pressure gradient within the boundary layer was stated to be constant, and identical with that of the outer flow. Moreover, a single parameter, the velocity gradient $a$, was used for characterization of the strain field, to which the flame is exposed. Application of experimental boundary conditions for both gas streams was not possible.

The new formulation presented in this paper estimates the pressure gradient within the boundary layer as an eigenvalue. No $a$ priori assumptions are made for the outer flow. Different types of boundary conditions can be specified at the free stream edge of the boundary layer. This allows for proper application of experimental boundary conditions to the calculations. As a result, calculated and mea- 
sured position and structure of the flame within the boundary layer shows a better agreement compared with the traditional approach.

Various laser-based measurements of velocities, temperatures and concentrations in a methane/air counterflow diffusion flame will be described and compared with calculations. Improved CARS measurements provided temperature profiles deduced from $\mathrm{N}_{2}$-Q-branch spectra. Two-dimensional concentration distributions of $\mathrm{OH}$ radicals were determined by $2 \mathrm{D}$-LIF using a tunable $\mathrm{KrF}$ excimer-laser. These distributions were calibrated to absolute concentrations with UV-absorption measurements in the A-X system of $\mathrm{OH}$. $\mathrm{OH}$ concentrations are especially important for comparison with reduced reaction schemes. ${ }^{12}$ Laser Doppler velocimetry with a two-color equipment was used to simultaneously determine the two components of the flow field.

\section{Experimental}

\section{Burner:}

The burner used in the experiments is similar in construction to the counterflow configuration used by Tsuji and coworkers. Compared to our set-up described previously ${ }^{10}$ this burner allows higher strain rates. Methane-regulated by flow controllers-was ejected from a porous brass cylinder (pore size $\approx 20 \mu \mathrm{m}$ ) glued to a water cooled cylindrical copper support of $40 \mathrm{~mm}$ diameter and a length of $100 \mathrm{~mm}$. The burner was installed in a vertically mounted wind tunnel with a square cross section at the burner head of $100 \times 100 \mathrm{~mm}$. Air was blown up the tunnel by four small fans.

\section{CARS Spectroscopy:}

A broadband CARS spectrometer has been installed which reduced the acquisition time by a factor of 30 compared to our previously used scanning CARS apparatus. ${ }^{11}$ The dye-laser and part of the $532 \mathrm{~nm}$ pump beam were collinearly combined in a USED-CARS phase matching geometry. The CARS signal was dispersed in a $1.3 \mathrm{~m}$-monochromator (McPherson Mod. 209) equipped with a 2400 $1 / \mathrm{mm}$ grating. The linear dispersion in the plane of the intensified diode array camera (SI, IRY 512) was measured to be $0.14 \mathrm{~cm}^{-1}$ per diode pixel. Averaged spectra (typically 300 laser shots) were stored and processed in a laboratory computer (SI, MfR III) or transferred to a larger computer (IBM 3090180) for further analysis.

Temperatures were deduced from computer generated least-squares-fitting spectral shapes of nitrogen vibrational-rotational Q-branch CARS spectra to measured spectra with temperature as a variable parameter. The distortion of the spectrum by the detector and spectrograph instrument functions, as well as the pump laser linewidth, had to be included in the simulation program by appropriate convolutions. Best results have been obtained by a priori fitting of these parameters to a CARS spectrum taken at room temperature. ${ }^{13}$ Concentration measurements of $\mathrm{N}_{2}, \mathrm{O}_{2}$ and $\mathrm{CH}_{4}$ were described elsewhere. ${ }^{14}$

\section{Two-Dimensional Laser-Induced Fluorescence:}

A tunable KrF-excimer laser (Lambda Physik EMG 150 TMSC) was used to excite single rotational lines of the $\left(v^{\prime}=3, v^{\prime \prime}=0\right)$ band in the $\mathrm{A}^{2} \Sigma^{+} \leftarrow \mathrm{X}^{2} \Pi$ system of $\mathrm{OH}$. Because of the rapid predissociation of the upper state the LIF-intensity is not affected by collisional quenching. ${ }^{\mathrm{I5}}$ The laser beam was focused to a light sheet with a cross section of $10 \times 1 \mathrm{~mm}$ and was sent through the flame along the stagnation line. A Nikon objective was used to image the fluorescence perpendicular to the light sheet onto a CCD-camera (Proxitronic Nanocam II). Gate times of $20 \mathrm{~ns}$ completely suppressed flame emission and daylight. ${ }^{16,17}$ Independent of the excitation wavelength, fluorescence of hydrocarbons was detected and could not be suppressed with filters (Schott UG 11, WG 295). Fluorescence images obtained from excitation on and off resonance with an $\mathrm{OH}$ transition were subtracted to obtain pure $\mathrm{OH}$ images. 100 single images were averaged to increase the signal-to-noise ratio. The population of the $\mathbf{N}^{\prime \prime}=8$ rotational state has only a small temperature dependence $(<10 \%)$ in the range under study. Therefore, 2D-LIF images obtained with $\mathrm{P}_{1} 8$ excitation directly show relative $\mathrm{OH}$ concentration distributions. 2D-temperature fields were determined by dividing the fluorescence intensities from successive $P_{1} 8$ and $Q_{1} 11$ excitation and calibrating one temperature point of the $2 \mathrm{D}$-field with a temperature determined with CARS. ${ }^{17}$

\section{UV-Laser-Absorption Spectroscopy:}

Absolute $\mathrm{OH}$ radical concentrations have been determined with UV-Laser absorption measurements. ${ }^{18}$ As shown in Fig. 1, a pulsed, frequency doubled dye laser (Lambda Physik FL 2002) pumped by an XeCl-exicimer laser (Lambda Physik EMG $201 \mathrm{MSC}$ ) was used to measure absorption spectra in the $\mathrm{A}^{2} \Sigma^{+} \leftarrow \mathrm{X}^{2} \Pi ;\left(v^{\prime \prime}=0, v^{\prime}=0\right)$ band of the $\mathrm{OH}$ radical. To achieve the spatial resolution necessary to resolve the flame structure, two pinholes (dia. $50 \mu \mathrm{m}$ ) were used. Straylight and flame emission were suppressed by Schott filters (UG 11) and short gate times of the boxcar integrator. Lines of the R-bandhead near $307 \mathrm{~nm}$ were recorded and synthetic spectra using published molecular data ${ }^{19,20}$ were fitted to these spectra to obtain rotational temperatures and absolute concentrations of $\mathrm{OH}$ 


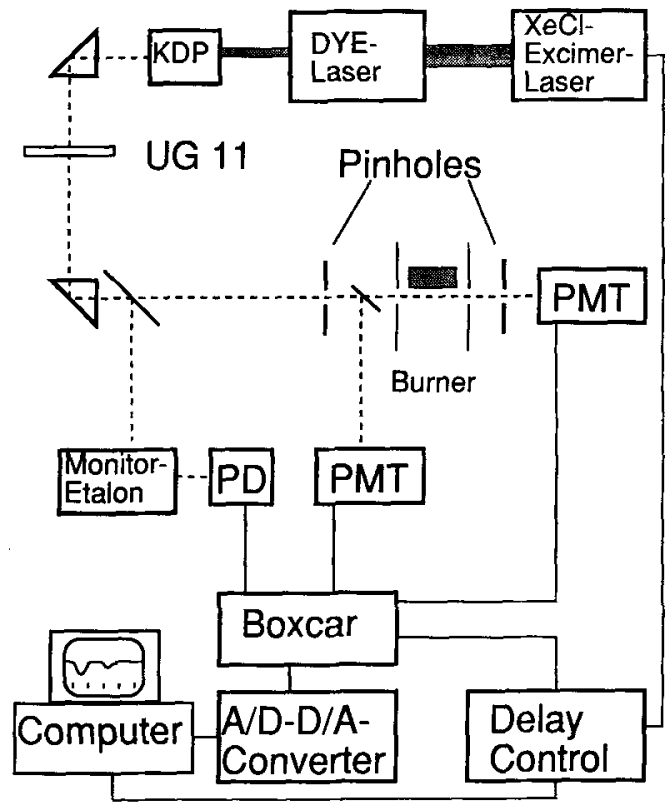

FiG. 1. Experimental arrangement for UV absorption spectroscopy of $\mathrm{OH}$ radicals with high spatial resolution in counterflow diffusion flames. (KDP: Frequency doubling crystal; PMT: Photomultiplier tube; PD: Photo diode).

radicals. The $\mathrm{OH}$ lineshapes were well described by a Voigt profile. Since the Voigt parameter $a$ depends on the unknown gas composition, the Lorentzian contribution to the profile was evaluated by the fitting routine. Changes of $\pm 15 \%$ in the Voigt parameter resulted in temperature and concentration changes smaller than $2 \%$. The laser profile was determined with a high resolution Fabry-Perot-interferometer (Burleigh RC 140) and was taken into account in the calculations.

\section{Laser-Doppler-Velocimeter and Data-Processing System:}

Laser-Doppler-Velocimeter measurements were performed with a two-channel dual beam LDV. The system arrangement is specially designed for the nonintrusive measurement in flames and two-phase flows. $^{21,22}$ The use of $\mathrm{MgO}$ powder as seeding particles is most convenient in this flow situation, as discovered in earlier work. ${ }^{23}$ The particles were added into the lower part of the wind tunnel with a controlled air flow (Tylan flowmeter). The 514.5 $\mathrm{nm}$ and the $488.0 \mathrm{~nm}$ lines of an Ar-Ion laser (Coherent Innova 90) are reflected separately into two parallel-mounted optical modules. To minimize the influence of refraction index fluctuation, the LDV system operates in the backscatter mode. With this arrangement single signals could be detected, which made it possible to detect the stagnation point. To distinguish counterflow direction and zero velocity, both channels are equipped with acousto-optic modulators (Bragg cells) to be able to downshift the signal frequency. The signals obtained, were converted and validated in Dantec counters and processed via a Dostek acquisition interface on a Compaq 286. Within the flame region and the stagnation point, comparison measurements were performed with a Dantec burst spectrum analyzer (BSA). Good agreement with both processing systems confirmed that distorted signals, originating from zones of low data rate, i.e. the flame front, did not affect the measured velocities.

\section{Model}

\section{Governing Equations:}

For a quantitative treatment of the flame structure the governing two-dimensional equations for overall-continuity, momentum, enthalpy, and mass of species $i$ are simplified traditionally by applying the boundary layer assumptions and by assuming an outer potential flow. In this way the problem is reduced spatially to one dimension, and the strain field to which the flame is exposed can be specified by the potential flow velocity gradient $a$. The pressure gradient within the boundary layer is assumed to be constant and is derived from the outer potential flow, ${ }^{9}$

To overcome certain deficiencies of the assumption of an outer potential flow, an alternative formulation is used. This formulation is based on ideas of Chapman and Bauer ${ }^{24}$ and Seshadri and Williams. ${ }^{25}$

To reduce the spatial order of the problem a stream function

$$
\psi(x, y) \equiv x \mathrm{~F}(y)
$$

identically satisfying the continuity equation is introduced. The boundary layer assumption allows one to treat density $\rho$, mass fractions of species $i w_{i}$, temperature $\mathrm{T}$, and the transport coefficients as functions of the radial coordinate $y$ only. The tangential velocity $u$ varies linearly with $x$, and the radial velocity $v$ is a function of $y$ only, too. The thermodynamic pressure $p$ is assumed to be constant within the flow field, with the pressure gradient still appearing in the momentum equations (small Mach number approximation).

Introduction of the stream function, application of the above assumptions to the two-dimensional governing equations, and resolving for the pressure gradients shows that the gradients $d p / d x$ and $d p /$ $d y$ are functions of $y$ only. Pressure $p$ is a state 
variable which implies that the mixed second derivatives being equal. Because both gradients are functions of $y$ only, the mixed second derivatives must be equal to zero. $d p / d y$ must be a constant, and can be estimated as an eigenvalue of the system. In the following this eigenvalue is abbreviated by $\mathrm{H}$ and $\mathrm{G}=d \mathrm{~F} / d y$ is introduced.

The modified boundary layer equations used for the description to the Tsuji counterflow configuration are:

Continuity

$$
\rho v=(\rho v)_{w}-\int_{0}^{y} \rho \mathrm{G} d y
$$

Momentum:

$$
\frac{\partial \mathrm{G}}{\partial t}=\frac{1}{\rho} \frac{\partial}{\partial y}\left(\mu \frac{\partial \mathrm{G}}{\partial y}\right)-\frac{\partial \mathrm{G}}{\partial y}-\mathrm{G}^{2}-\frac{\mathrm{H}}{\rho}
$$

Temperature:

$$
\begin{aligned}
\frac{\partial \mathrm{T}}{\partial t}=\frac{1}{\rho c_{p}} \frac{\partial}{\partial y}\left(\lambda \frac{\partial \mathrm{T}}{\partial y}\right)-v \frac{\partial \mathrm{T}}{\partial y} & \\
& -\frac{1}{\rho c_{p}} \sum_{i} c_{p, i} j_{i} \frac{\partial \mathrm{T}}{\partial y}-\frac{1}{\rho c_{p}} \sum_{i} h_{i} r_{i}
\end{aligned}
$$

Mass of species $i$ :

$$
\begin{aligned}
\frac{\partial w_{i}}{\partial t}=\frac{1}{\rho} \frac{\partial}{\partial y}\left(\mathrm{D}_{i}^{m} \frac{\partial w_{i}}{\partial y}\right) & -v \frac{\partial w_{i}}{\partial y} \\
& +\frac{1}{\rho} \frac{\partial}{\partial y}\left(\mathrm{D}_{i}^{\mathrm{T}} \frac{\partial \ln \mathrm{T}}{\partial y}\right)+\frac{r_{i}}{\rho}
\end{aligned}
$$

$(t=$ time, $v=$ flow velocity in $y$-direction, $y=$ cartesian space coordinates, $p=$ pressure, $\mu=\mathrm{dy}$ namic viscosity, $c_{p}=$ specific heat capacity, $\lambda=$ heat conductivity, $r=$ mass scale chemical rate of formation, $j_{i}=$ diffusive mass flux, $D_{i}^{m}=$ modified binary diffusion coefficient, $\mathrm{D}_{i}^{\mathbf{T}}=$ thermodiffusion, index $i$ stands for the $i$ th chemical species of the system).

The boundary conditions at $y=0$ are given by

$$
\begin{aligned}
\rho v & =(\rho v)_{w} \\
\mathrm{G} & =0 \\
\mathrm{~T} & =\mathrm{T}_{w} \\
w_{i} & =w_{i, u}-j_{i, w} /(\rho v)_{w}
\end{aligned}
$$

Because of the recombination of $\mathrm{H}$-atoms at the cylinder wall surface, mass fractions for these atoms are handled differently:

$$
\begin{aligned}
w_{\mathrm{H}} & =0 \text { and } \\
w_{\mathrm{H}_{2}} & =w_{\mathrm{H}_{2, u}}-j_{\mathrm{H}_{2}, w} /(\mathrm{p} v)_{w}-j_{\mathrm{H}, w} /(\rho v)_{w}
\end{aligned}
$$

(index $w$ denotes conditions at the cylinder, index $u$ denotes conditions of the unburnt gas). The respective boundary conditions at the outer edge of the flame $y_{e}$ are

$$
\begin{aligned}
\partial \mathrm{G} / \partial y & =0 \\
\mathrm{~T} & =\mathrm{T}_{e} \\
w_{i} & =w_{i, e} \quad i=1,2, \ldots, \mathrm{N}
\end{aligned}
$$

(index $e$ denotes conditions of the free up-streaming gas).

Equations (3) to (5) are transformed into finite difference expressions by a parabolic discretization in space and a linear one in time ${ }^{26}$ At the beginning of the integration, 40 grid points are non-uniformly spread across the flame front, with a distribution adapted to the temperature profile gradient. For educts, cubic polynomials, for temperature, intermediate species, and for products, Gaussian profiles are chosen as starting estimates.

Due to stiffness of the system considered, an implicit solution method is used ${ }^{27}$ During the integration, the grid point system is statically adapted according to a weighted norm of gradients of temperature and some species profiles. ${ }^{28}$ Interpolation onto the new mesh is performed by piecewise monotonic cubic hermite interpolation. ${ }^{29}$

\section{Transport Processes, Reaction Mechanism and Thermochemistry:}

For the calculation of diffusion coefficients $D_{i}^{M}$, thermal diffusion coefficients $\mathrm{D}_{i}^{\mathrm{T}}$, heat conductivities $\lambda$, and dynamic viscosities $\mu$, data are taken from molecular parameters. To calculate the transport parameters, formulae given by Kee et al. are used. $^{30}$

A simplified transport model is used, because comparison with multicomponent transport models leads to errors that are relatively small compared to those caused by uncertainties in the reaction mechanism. ${ }^{31}$

The reaction mechanism consists of 250 elementary steps (including reverse reactions), and of 39 chemical species. The $\mathrm{H}_{2}-\mathrm{O}_{2}$-system, $\mathrm{CO}$ oxidation, degradation of methane to $\mathrm{CO}$, alkyl recombination to ethane, and its consumption reactions are included. ${ }^{9}$ Kinetic data are taken as in ${ }^{10}$ with minor revisions due to results of a new evaluation of data. ${ }^{32}$

\section{Results and Discussions}

Each of the experimental methods had a spatial resolution of better than $60 \mu \mathrm{m}$, thus the flame zone 
was well resolved. The resolution of the CARS measurements $(60 \mu \mathrm{m})^{10}$ and the absorption measurements $(50 \mu \mathrm{m})$ was verified with a knife edge moved perpendicular to the laser beam while observing the signal intensity. The $2 \mathrm{D}$-LIF measurements have a transversel resolution of approximately $10 \mu \mathrm{m}$, depending on the magnification of the lens. A millimeter scale was recorded with the CCD-camera and gave the length scale for the 2D images. The resolution achieved with the LDV-system is about $30 \mu \mathrm{m}$.

One-dimensional LDV measurements in the symmetry plane of the flame yielded velocity profiles up to the stagnation point with the typical shape reported by Tsuji et al. ${ }^{13}$ As the burner geometry does not allow optical access up to the burner head with the two channel LDV arrangement, the absolute $y$ position of the $2 \mathrm{D}$ flow field was scaled with the position of one-dimensional velocity profiles. The calculations performed earlier ${ }^{9}$ used the velocity gradient $a$ for the characterization of the strain field. This implies that the flow field can be described as an outer potential flow surrounding the boundary layer. However, the measured flow field in Fig. 2 shows that the divergence of the stream lines can not be described as a parallel or potential flow. So the assumption of a potential flow is not valid for the description of this flame. In the new model, the input of experimental velocity data allows a better treatment of the real flow situation. Comparisons with calculations show that the theo-

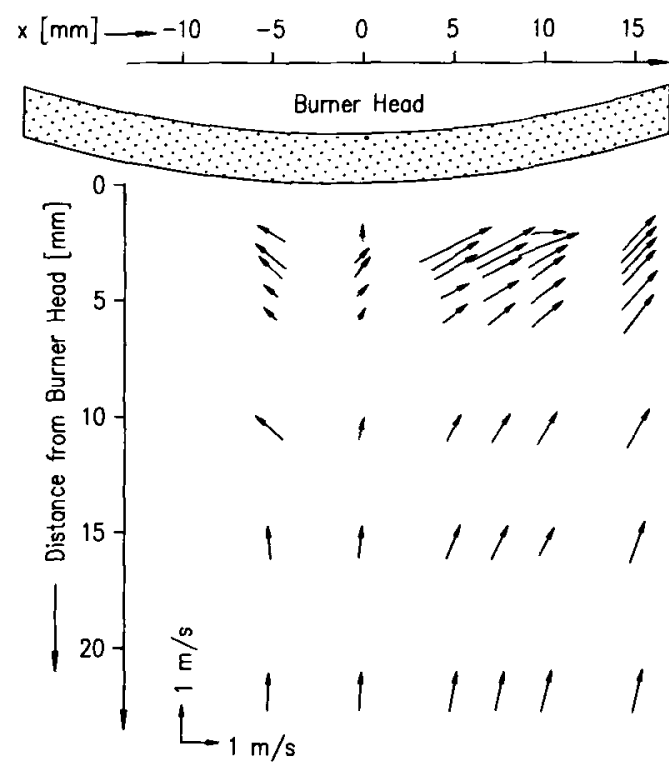

FIG. 2. Flow field in the forward stagnation region of a counterflow diffusion flame $\left(a=125 \mathrm{~s}^{-1}\right.$, $v_{u}=11,4 \mathrm{~cm} / \mathrm{s}$ ) measured with a two-channel dual beam Laser Doppler Velocimeter. retical profiles are located too far on the air side of the flame. This is also observed for temperature and concentration profiles. The experimental temperature profiles (CARS, Absorption and 2D-LIF) differ in location of their peaks. The difference is smaller than $0.5 \mathrm{~mm}$ and is due to a refractive index change across the flame. As observed experimentally, this effect is smaller for light beams perpendicular to the cylinder axis. Nevertheless, relative corrections of the positions as described earlier ${ }^{10}$ are unaffected of this procedure. Therefore the absolute positions from 1D-LDV and 2D-LIF were used as standard. The absorption and CARS measurements have been shifted to the positions determined with 2D-LIF. Figure 3 shows calculated and measured temperatures for a flame with $a=125 \mathrm{~s}^{-1}$. The parameter $a$ is used to give an approximate comparison with older measurements. CARS- $\mathbf{N}_{2}$-temperatures are taken for comparison with calculations, because $\mathrm{OH}$ radicals are found in a small spatial region only, so $\mathrm{N}_{2}$ is the better temperature indicator in this case. The peak rotational temperature of $\mathrm{OH}$ was calculated from absorption spectra to be $1910 \mathrm{~K}$, com pared with $1919 \mathrm{~K}$, measured with CARS. Each of these temperatures $( \pm 40 \mathrm{~K})$ is lower than the 1969 $\mathrm{K}$ predicted by the calculations.

For the $\mathrm{OH}$ concentration measurements, the effective absorption pathlength, which varies with distance from the bumer head was determined with 2D-LIF. Part of the $\mathrm{OH}$ distribution is shown in Fig. 4. Approaching the edges of the cylinder, the flame is bent slightly towards the cylinder surface. The bent zones are short and sharp, as observed by imaging $\mathrm{OH}$ concentrations and temperatures with 2D-LIF. Deviations from one-dimensionality can be neglected because the fits of the absorption spectra show no deviations from Boltzmann distributions and the rotational temperatures are con-

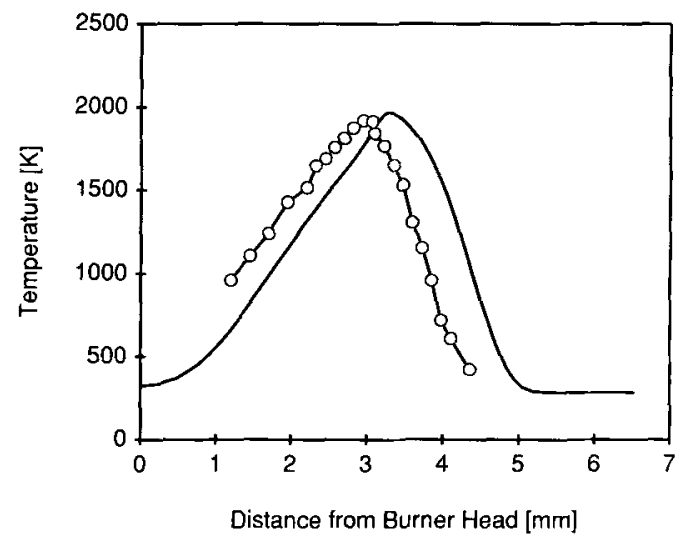

FIG. 3. Temperature profile in a counterflow diffusion flame $\left(a=125 \mathrm{~s}^{-1}, v_{u}=11,4 \mathrm{~cm} / \mathrm{s}\right) ; 0$ CARS measurement - Simulation 

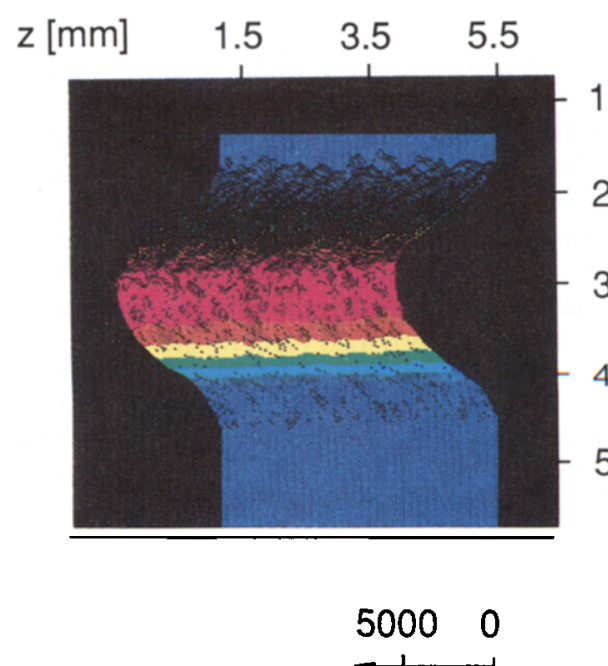

ppm [OH]

Fic: 4. Two-dimensional distribution of the absolute $\mathrm{OH}$ radical concentration in a counterflow diffusion flame $\left(a=125 \mathrm{~s}^{-1}, v_{u^{\prime}}=11,4 \mathrm{~cm} / \mathrm{s}\right)$, imaged after excitation of the $P_{1} 8$-line of the $\left(v^{\prime}=3\right.$, $\mathbf{u}^{\prime \prime}=0$ ) band of the A-X-system with a tunable $\mathrm{KrF}$ excimer laser, detected with an image-intensified CCD camera and calibrated with absorption measurements

sistent with CARS temperatures. ${ }^{33}$ The absolute concentration profile on Fig. 5 was obtained by calibrating relative concentrations from $2 \mathrm{D}$-LIF measurements with the peak concentration of the absorption measurements. Again, the peak position, predicted by the model is too far on the air side of the flame. The experimental $O H$ radical concentration is higher than the concentration calculated with the model. This discrepancy cannot be explained by experimental uncertainty $(\approx 10 \%)$.

Detailed measurements of temperatures, concentrations and velocities in a counterflow diffusion flame have yielded an important basis set of experimental data for comparison with mathematical flame models. Although the model has been improved, there are still differences between experiment and calculations. Knowledge of OH radical concentrations is especially important in optimizing mathematical flame models, because the measured temperature profile can be modeled very well, whereas differences still remain in the OH concentrations. Earlier calculations ${ }^{9}$ predicted $\mathrm{OH}$ concentrations in good agreement with our measured values but as mentioned, the parameter a can not describe the flow situation, so the results are not comparable. Results of calculations by Puri et al., ${ }^{3.1}$ which are also close to our experimental values, describe a

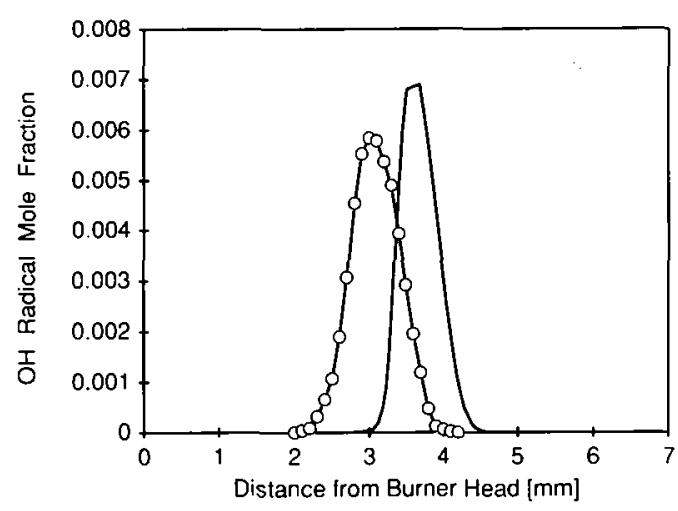

FIG. 5. Absolute OII radical concentration profile in a counterflow diffusion flame $\left(a=125 \mathrm{~s}^{1}, v_{x}=\right.$ $11,4 \mathrm{~cm} / \mathrm{s}): \bigcirc$ Absorption \& Light sheet measurements, - Simulation

different flow situation and no experimental data are available for this flame. The differences between cxperiment and calculations may be due to shortcomings of the transport model, specially for small molecules. From the experimental point of view the counterflow flame arrangement is thought to be an appropriate tool for the development of two-dimensional diagnostic techniques because of the special flame structure and the variety of studies performed on the structure of this type of flame.

\section{Acknouledgment}

The financial support of the Bundesininsterium für Forschung und Technologie for the Research Association TEC:FLAM under contract No. 03 E6325B is gratefully acknowledged.

\section{REFERFNCES}

1. Willasys, F. A.: "Turbulent mixing in Non-reactive and Reactive Flows," p 189, (S.V.B. Murphy. Ed.), Plenum, 1975.

2. Bray, K. V. C.: Springer Ser, in Chem. Phys. (J. Warnatz. WV. Jäger, lids.) 47, 356 (1987).

3. PETtiks. N.: Twenty-rirst Symposium (International) on Combustion, p. 1231. The Combustion Institute, 1988.

4. Tsujl. H.: Progr. Fnergy Comb. Sci. 8, 9.3 (1982).

5. Tsijl, H., YanaokA, I.: Eleventh Symposium. (International) on Combustion, p. 979, The Combustion Institute. 1967.

6. Ts(J), H. Yanaoka, I.: Twelfth Symposium (International) on Combustion, p. 997, The Combustion Institute, 1969. 
7. TsujI, H., Yamaoka, I.: Thirteenth Symposium (International) on Combustion, p. 723, The Combustion Institute, 1971.

8. Yamaoka, I., TsujI, H.: Fifteenth Symoposium (International) on Combustion, p 637, The Combustion Institute, 1975.

9. Dixon-Lewis, G., David, T., Gaskell, P. H., Fukutani, S., Jinno, H., Miller, J. A., KeE, R. J., Smooke, M. D., Peters, N., Effels. BErG, E., Warnatz, J., Behrendt, F.: Twentieth Symposium (International) on Combustion, p. 1893, The Combustion Instititute, 1985.

10. Dreier, T., Lange, B., Wolfrum, J., ZahN, M., Behrendt, F., Warnatz J.: Twenty-First Symposium (International) on Combustion, p. 1729, The Combustion Institute, 1988.

11. Dreier, T., Lange, B., Wolfrum, J., Zahn, M., Behrendt, F., Warnatz, J.: Ber. Bunsenges. Phys. Chem, 90, 1010 (1986).

12. Paczko, G., Lefdal, P. M., Peters, N.: TwentyFirst Symposium (International) on Combustion, p. 739, The Combustion Institute, 1988.

13. Dießel, E., Dreier, T., Lange, B., Wolfrum: Appl. Phys. B50, 39 (1990).

14. Dreier, T., Lange, B., Wolfrum, J., Zahn, M.: Appl. Phys. B45, 183 (1988).

15. Andresen, P., Bath, A., Gröger, W., Lülf, H. W., Meijer, G., ter Meulen, J. J.: Appl. Optics 27, 365 (1988).

16. Suntz, R., Beckek, H., Monkhouse, P., WolFrum, J.: Appl. Phys. B 47, 287 (1988).

17. Arnold, A., Becker, H., Ketterle, W., Monkhouse, P., Suntz, R., Köllner, M., Wolfrum, J.: VDI-Berichte, Nr. 765, 457 (1989).

18. Sмyтн, К. С., Тرossem, P. J. H., Hamins, A., Miller, J. H.: Flame, 79, 366 (1990).

19. Dieke, G. H., Crosswhite, H. M.: J. Quant. Spectr. Rad. Transf. 2, 97 (1962).

20. Goldman, A., Gillis. J. R.: J. Quant. Spectr. Rad. Transf. 25, 111 (1981).

21. Thiele, K.-U., Brodbeck, H.: Selected Papers of the 3rd Intern. Symposium Application of LDA to Fluid Mechanics p. 513, Lisbon, 1987.
22. ThIELE, K. -U.: 6th International Congress on Applications of Laser and Electro-Optics p. 21, San Diego, USA, 1987.

23. Thiele, K. -U., Brodbeck, H.: International Flame Research Foundation, 9th Members Conference, Noordwijkerhout, Netherlands, 1989.

24. Chapman, T. W., Bauer, G. L., Appl. Sci. Res. 31,223 (1975).

25. Seshadri, K., Williams, F. A.: Int. J. Heat Mass Transfer, 21, 251 (1978).

26. Warnatz, J.: Ber. Bunsenges. Phys. Chem. 82, 193 (1981).

27. Forsythe, G. E., Wasow, W. R.: Finite-Differences-Methods for Partial Differential Equations, Wiley, New York, 1960.

28. KAUTSKY, J., NiChOLS, N. K.: Equidistributing Meshes with Constraints, SIAM J. Sci. Stat. Comput., 1, 449 (1980).

29. Fritsch, F. N., Carlson, R. E.: SiaM J. Numer. Anal. 17, 238-246 (1980).

30. Kee, R. J., Warnatz, J., Miller, J. A.: A FORTRAN Computer Code package for the Evaluation of Gas-Phase Viscosities, Conductivities and Diffusion Coefficients, Sandia National Laboratories Report SAND 83-8209, Livermore, USA 1983.

31. Warnatz, J.: Influence of Transport Models and Boundary Conditions on Flame Structure, Numerical Methods in Laminar Flame Propagation (Peters N., Warnatz J. Eds), Friedr. Vieweg \& Sohn, Braunschweig/Wiesbaden, 1982.

32. Baulch, D. L., Just, T., Kerr, J. A., Troe, J., Walker, R. W., Wahnatz, J.: "Compilation of Rate Data on $\mathrm{Cl} / \mathrm{C} 2$ Species Oxidation," J. Phys. Chem. Ref. Data, publication in preparation 1990.

33. Rensberger, K. J., Jeffries, J. B., Copeland, R. A., Kohse-Hölnghaus, K., Wise, M. L., Crosley, D. R.: Appl. Opties 28, 3556 (1989).

34. Puri, I. K., Seshadri, K., SMooke, M. D., Keyes, D. E.: Combust. Sci. and Tech., 561 (1987). 